\title{
Possibility of Assessing the Gastrointestinal Tract as a Complex System
}

\author{
Roham Mazloom ${ }^{1}$ \\ Received: 23 January 2019 / Accepted: 30 January 2019 / Published online: 7 February 2019 \\ (C) Springer Science+Business Media, LLC, part of Springer Nature 2019
}

\section{Dear editor:}

I am interested in Dariusz Komorowski paper entitled "EGG DWPack: System for Multi-Channel Electrogastrographic Signals Recording and Analysis". Electrogastrography (EGG) and electrogastroentrography (EGEG) detect slow waves and myoelectrical activity of the gastrointestinal (GI) system from the surface of the abdominal skin using power spectral analysis. Moreover, EGG DWPack software which introduce by Komorowski analyzes the linear indices of heart rate variability (HRV) simultaneously. This method analyzes the frequency and power of GI electrical waves as well as HRV linear parameters. Although slow waves are subject to alteration depending on the condition of the GI, yet spectral analysis of EGG and EGEG cannot be used for diagnosing specific GI diseases, since they are non-specific tests, thus making them controversial for clinical use.

On the other hand, the GI system is formed of numerous organs and tissues communicating through a network of hormonal and nervous systems. Thus, the connections between different parts of the GI system are complicated. The GI tract demonstrates the properties of complex systems. Furthermore, the syncytium structure of GI cells creates even more complexity. This connectivity contributes to the spontaneous or- der, self-organization, and adaptation of the GI system. It seems that variability of GI electrical slow waves also demonstrates the dynamics of complex systems. This approach may help us to use other methods for study electrical activity of the GI system more than frequency and power of slow waves.

Several methods, such as non-linear analysis, can be put to use in studying the GI tract if we consider it a complex system. Nonlinear approaches help us clarify the dynamics of EGG and EGEG variations. Peak-to-peak intervals can be obtained once slow wave peaks have been detected. Correlations between peak-to-peak intervals reveal the dynamics of slow waves. Several non-linear methods can be used in order to assess the various dimensions of slow wave dynamics, including, short-term and long-term variation, fractal structure, entropy, and chaotic characteristics. Nonlinear analysis of slow wave dynamic variations, if used in parallel with power spectral analysis, may help discern different GI system diseases from one another. However, nonlinear approaches towards GI slow waves should be investigated in physiologic and pathologic conditions.

Publisher's Note Springer Nature remains neutral with regard to jurisdictional claims in published maps and institutional affiliations.
This article is part of the Topical Collection on Image \& Signal Processing

Roham Mazloom

rohammazloom@abzums.ac.ir

1 Department of Physiology-Pharmacology-Medical Physics, Alborz University of Medical Sciences, Karaj, Alborz, Iran 